\title{
IR RECOMBINATION LASERS
}

\author{
W. J. Witteman, P. J. M. Peters, H. Botma and Y. B. Udalov \\ Department of Applied Physics, University of Twente, P.O. Box 217, 7500 AE Enschede, \\ The Netherlands
}

(Received 30 May 1994)

\begin{abstract}
The present study contributes to a better understanding of the atomic Xe laser as a powerful IR source. Several important phenomena like the dependence of both the observed optimized input power and maximum output power on the square of the gas density and also the constant fractional ionization are reported and verified theoretically. The insight in the kinetics of this system has also lead to the realization of small-size continuous systems with output powers in the range of watts. The results of the present study can be used to predict the performance of the atomic Xe laser under different operating conditions.
\end{abstract}

\section{INTRODUCTION}

Lasing from neutral Xe atoms excited in a gas discharge was observed for the first time in the early 1960 s, shortly after the invention of the laser. The beginning of the laser era was characterized by an extensive search for "anything that could lase." Most of the new laser systems did not receive continuous interest because of their low power and have been forgotten. They may remain interesting for the history of science. This was not the case for the neutral Xe laser although it was close to this fate. This laser never went out of the scene just because of one unique feature, i.e. an extremely high gain in a very homogeneous $\mathrm{cw}$ gas discharge. It has been observed that a gain as high as $1000 \mathrm{~dB} / \mathrm{m}$ is feasible with a low pressure (hundreds of millitorr to a few torr) discharge in $\mathrm{He}-\mathrm{Xe}$ mixtures. The disappointment of this conventional system is that along with the extremely high gain the atomic Xe laser has an extremely low saturation intensity. As a result it appeared to be a laser that could operate practically without mirrors but even with optimum outcoupling the output power was very small typically in the order of a few hundreds of microwatts with an efficiency far below $1 \%$.

\section{BREAKTHROUGH IN THE DEVELOPMENT OF HIGH POWER IR LASERS}

Nevertheless, research interest in this laser has not weakened; in fact it has been strengthened over the past $5 \mathrm{yr}$. The reason is that this laser is now becoming the most promising light source in the wavelength region of a few microns when using a high-pressure rare gas mixture with less than $1 \% \mathrm{Xe}$. Its major advantages are high efficiency (so far up to $8 \%$ ), high output energies $(15 \mathrm{~J} / 1$ at 9 bar pressure), high continuous output power (more than $200 \mathrm{~W} / \mathrm{l}$ ), no gas dissociation and no thermal heating of the lower laser level.

The major breakthrough in this type of laser was possible after the technology for e-beam and e-beam sustained systems and later the technology for pulsed high-pressure discharges with UV and $x$-ray preionization became available. Especially the e-beam sustained technology which was successfully developed during the 1980s for high-energy excimer systems is crucial for obtaining a high performance in pulsed operation. The important point by applying these very different 
discharge technologies is the possibility to expand the range of working pressures substantially which turns out to be essential for obtaining higher power and better efficiency. From these observations and the accompanying theoretical modeling the idea was supported to consider a multi-atmosphere Xe laser as a four-level system that uses the metastable state of the lasing atom as the ground state in the excitation scheme. ${ }^{(1)}$ The primary channel for filling of the upper laser level in the Ar-Xe mixture is the dissociative recombination of $\mathrm{ArXe}^{+}$or $\mathrm{Xe}_{2}^{+}$molecular ions with electrons. After the ions recombine they decay to form the upper laser level, hence the name "Recombination laser." The lower level is emptied in collissions with atoms of the buffergas Ar and with electrons by decaying to the metastable level. The atoms in the metastable state are then ionized again in the discharge. The high lasing efficiency is a consequence of the fact that the quantum efficiency of the Xe laser increases because of a recirculation of energy without a relaxation of metastable atoms to the ground state. Ionization from this metastable state requires only $3.8 \mathrm{eV}$. See Fig. 1 .

The lasing of atomic Xe occurs on the well-known IR transitions $(1.73-3.51 \mu \mathrm{m})$ between the $5 \mathrm{~d}$ and $6 \mathrm{p}$ manifolds as shown in Fig. 2 . The $5 \mathrm{~d}$ manifold is efficiently populated through the recombination process with a resulting quantum efficiency of $19 \%$ for the $1.73 \mu \mathrm{m}$ line. Although high power operation is obtained the system saturates with increasing electron density i.e. current density. The mixing of the $5 \mathrm{~d}$ and $6 \mathrm{p}$ manifolds by the electrons is always in competition with the stimulated emission process of the laser. With increasing pump power, as we shall see later on, the mixing process wins at the expense of stimulated emission. As a result both lasing efficiency and lasing power decrease above a certain input power level.

The above-mentioned recombination is a three-body-collision process. It is the principal mechanism in the inversion process. This can only occur at high gas density, say above 100 Torr. The increased pressure leads also to homogeneous line broadening which has a beneficial effect on the efficiency. However, a homogeneous inversion density under such conditions requires a homogeneous discharge in a high-density gas. This is from a technical point of view a great challenge. A self-sustained discharge at these densities is in principle unstable. Moreover, at high gas density the pressure broadening mechanism of the laser transitions reduces the gain. At a constant inversion density the gain is inversely proportional to the gas density, whereas the saturation intensity increases proportionally to this density. In order to maintain sufficient gain to reach saturation in a laser system it is necessary to also increase the inversion density proportional to the gas density. This means, however, that in a laser volume of, say, $100 \mathrm{~cm}^{3}$ at $1 \mathrm{~atm}$ gas pressure the input power is of the order of $250 \mathrm{~kW}$. At first glance this is not realistic for continuous operation. For that reason most attention is paid to pulsed systems where during the pulse the input energy is sufficiently high and the average power is set by the pulse repetition frequency. The

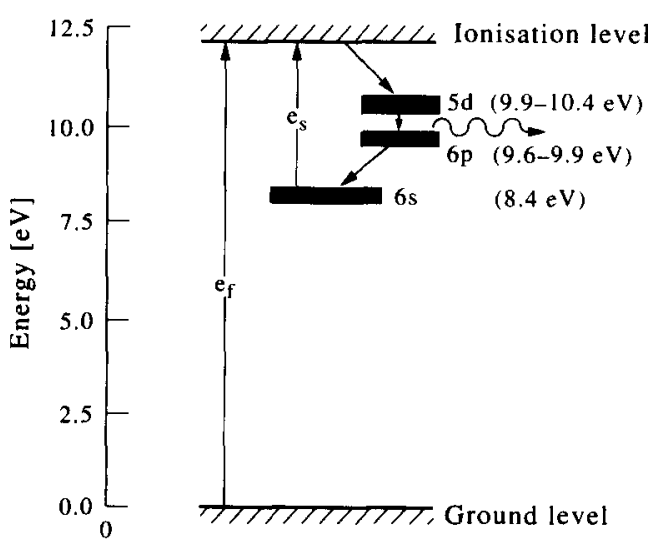

Fig. 1. Four-level scheme of the atomic Ar-Xe laser. 


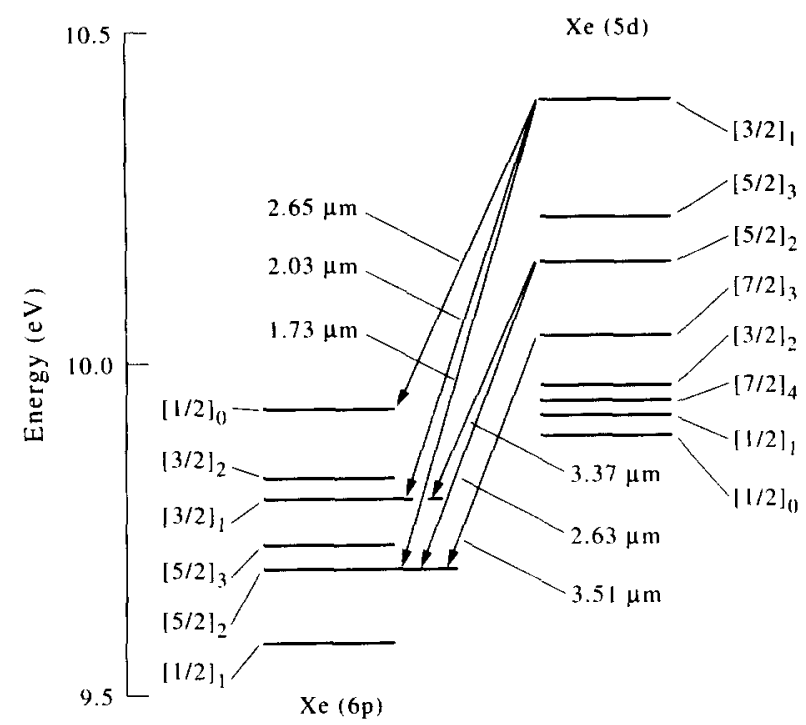

Fig. 2. Energy levels of the $5 \mathrm{~d}-6 \mathrm{p}$ manifolds of Xe showing the laser transitions.

above-mentioned technology for pulsed discharges at high-pressure has been applied in a modified version to investigate this NIR atomic laser. Stable short pulse discharges are either $\mathbf{U V}^{(2)}$ or $\mathrm{x}$-ray ${ }^{(3.4)}$ preionized, electron beam pumped ${ }^{(1)}$ or electron beam sustained. ${ }^{(1.5)}$

\section{CONTINUOUS OPERATION WITH OUTPUT POWER IN THE RANGE OF WATTS}

In spite of the high gas density requirement the search for making cw systems with significant output power has been continued. Various discharge configurations were investigated. Unfortunately, most attempts were in vain, especially those based on a direct current discharge (d.c.). One approach to investigate discharge stability with increasing pressure is to narrow the discharge channel according to the similarity rules which state that pressure times discharge diameter is constant. From our research on pulsed mode operation we obtained, as will be discussed later on, that the required input power density is about $1-2.5\left(\mathrm{~kW} \mathrm{~cm} / \mathrm{atm}^{2}\right)$. This means that for a narrow channel of about $0.1 \mathrm{~cm}^{2}$, an active length of $50 \mathrm{~cm}$ and a gas pressure of 100 Torr about $100-250 \mathrm{~W}$ input power is required in order to make full profit of the above-mentioned laser mechanism. This requirement is not easy to fulfil, especially because of the high gas density. Even in very narrow capillaries of $1 \mathrm{~mm}$ dia the maximum attainable gas density is still too low for a substantial increase in output power in the case of d.c. discharge. Since discharge stability and homogeneity are in general better ensured with a radio frequency (RF) discharge than with a normal d.c. discharge we successfully explored RF excitation for this lasing gas system as a function of gas pressure. ${ }^{(6)}$ This technique has several benefits. It operates as a transverse discharge between a narrow gap of two parallel electrodes so that a relatively low discharge voltage is sufficient and good spatial homogeneity is obtained. The free choice of the excitation frequency gives an extra parameter to control the gas discharge. A particular advantage to the recombination laser pumping is that the rise and, more importantly, the fall times of the RF-excitation are independent of the discharge electrical parameters. We obtained uniform $\mathrm{RF}$ excited gas discharge plasmas in various laser resonator geometries including slab systems. So far we obtained for the atomic Xe laser with RF excitation at $110 \mathrm{MHz}$ a cw output power of $1.5 \mathrm{~W}$ in a slab configuration with a cross-section of $2.25 \times 8 \mathrm{~mm}^{2}$ and an active length of $37 \mathrm{~cm}$, using a gas mixture $\mathrm{Ar}: \mathrm{He}: \mathrm{Xe}=60: 39: 1$ at a total pressure of 100 Torr. The input power is about $300 \mathrm{~W}$. 


\section{THEORETICAL MODEL OF THE LASING PROCESS}

The dynamic behaviour of the laser can be described by a set of coupled rate equations. In a relatively simple approach a set of simultaneous differential equations describes the population inversion and the radiation density within a spatially uniform laser medium. We will describe the system in terms of the energy level diagram shown in Fig. 1. As we have seen above, two manifolds of energy levels, $5 \mathrm{~d}$ and $6 \mathrm{p}$, are of prime importance in this laser. Since each laser line has the upper laser level in the $5 \mathrm{~d}$ and the lower level in the $6 \mathrm{p}$ manifold and since the distributions within the manifolds are not important for the total output of the laser, we consider each manifold as an average energy level: the $5 \mathrm{~d}$ manifold is the upper laser level $E_{2}$ with density $N_{2}$ and the manifold $6 \mathrm{p}$ the lower laser level $E_{1}$ with density $N_{1}$. For our analysis of the laser process an approximation of the four-level system as indicated in Fig. 1 by a two-level representation is very useful.

The rate equation approach we will then follow involves a number of simplifying assumptions; in using a single set of rate equations we will ignore longitudinal and radial variations of the radiation within the laser. In spite of these limitations our simple rate-equation approach remains a useful tool and provides us a great deal of insight into the behaviour of this recombination laser. We will derive from these rate equations an expression for the maximum output power as it depends on the gas density. Also the optimized relation between the electron density and gas density, the so-called fractional ionization, is obtained. Furthermore with the results we will argue the observed differences in the performances of e-beam systems, e-beam sustained systems and self-sustained $x$-ray preionized systems. On the other hand, many details of the laser performance, like the descriptions of the resonant modes and the spectral distribution of the laser emission are not included in the model. However, these details can be accounted for independently.

The rate of change of the photon density $\rho$ (photons $/ \mathrm{cm}^{3}$ ) within the resonator can be described by:

$$
\frac{\mathrm{d} \rho}{\mathrm{d} t}=c \rho \sigma\left(N_{2}-N_{1}\right)-\frac{\rho}{\tau_{\mathrm{cav}}}
$$

where $\sigma$ is the cross-section for stimulated emission and $\tau_{\mathrm{cav}}=2 l / c(1-R)$ is the decay time for photons in the optical resonator. For stationary conditions the inversion is given by

$$
N_{2}-N_{1}=\frac{1}{c \sigma \tau_{\text {cav }}}
$$

The densities $N_{2}$ and $N_{1}$ are both quenched by electrons and heavy particles. These quenching collisions reduce the inversion by coupling the upper $5 \mathrm{~d}$ laser level to the lower $6 \mathrm{p}$ level. The occupation of the levels have the tendency to thermalize by the collisional coupling and thus to destroy the inversion. With respect to the electrons we have the situation that electrons recombine with ions to produce upper laser states and on the other hand the electrons quench the inversion. Similarly the higher the gas density the more ions can be produced and on the other hand, the more of the inversion is quenched by the gas density. So it is expected that for both electrons and atoms there are optimized densities for which the laser output is maximum.

We observed for the discharge of this laser that the electric field $E$ is practically independent on the discharge current density $i$. The electric field in turn is found to be proportional to $N$, so that $i$ is proportional to $P / N$ where $P$ is the power deposition density and $N$ the gas density. Further, the drift velocity $v_{D}$ which is proprotional to $E / N$ is constant so that the electron density $n$ is proportional to $i$. Therefore the quenching of the upper state by the electrons is given by $q^{\prime} N_{2} P / N$ where $q^{\prime}$ is a constant of proportionality. Although the collision mixing affects both laser levels it can be argued that because of the fixed relation between the two levels as given by (2) the effective loss of the upper states is given by the last expression where we replace $q^{1}$ by $q$. Similarly the 
quenching of the upper laser level by the atoms is proportional to $N$ and given by $p N_{2} N$ where $p$ is also a constant of proportionality.

The change in the population density of the upper laser level is then given by:

$$
\frac{\mathrm{d} N_{2}}{\mathrm{~d} t}=\alpha P-c \rho \sigma\left(N_{2}-N_{1}\right)-\frac{q N_{2} P}{N}-p N_{2} N,
$$

where the production term on the right-hand-side is proportional to $P$. The radiative decay of the upper state can be neglected in comparison with the other processes. Similarly the rate equation for the lower laser level becomes:

$$
\frac{\mathrm{d} N_{1}}{\mathrm{~d} t}=\beta P+c \rho \sigma\left(N_{2}-N_{1}\right)+\frac{q N_{2} P}{N}+p N_{2} N-\frac{N_{1}}{\tau},
$$

where $\beta P$ is a production term and $\tau$ is the lifetime of the lower state. This lifetime is determined by radiation and collisional relaxation to other states. For both processes it is noticed that at higher pressures collisions with the gas atoms limit the lifetime. Considering stationary conditions we obtain from (3) and (4):

$$
P(\alpha+\beta)=\frac{N_{1} N}{\tau_{0}}
$$

where we used $\tau_{0}=N \tau$ since the lifetime is inversely proportional to density $N$. In practice $N_{2}-N_{1}$ as given by (2) is much smaller than $N_{2}$ so that we may replace $N_{1}$ by $N_{2}$ in (5). Substituting the last result in (3) and making use of (1) we find for the output power $W=h v \rho / t_{\text {cav }}$ per unit volume:

$$
W=h v\left\{\alpha-(\alpha+\beta) p \tau_{0}\right\} P-h v(\alpha+\beta) q \tau_{0} \frac{P^{2}}{N^{2}} .
$$

We see that for small values of $P$ the output is proportional to the input, independent of $N$. The optimized input power density $P_{\mathrm{opr}}$ for which the output $W$ is maximum is obtained from $\mathrm{d} W / \mathrm{d} P=0$. We find:

$$
P_{\mathrm{opt}}=\frac{N^{2}}{2(\alpha+\beta) q \tau_{0}}\left\{\alpha-(\alpha+\beta) p \tau_{0}\right\}
$$

Substituting (7) into (6) we find the maximum power $W_{\max }$ :

$$
W_{\max }=\frac{h v\left\{\alpha-(\alpha+\beta) p \tau_{0}\right\}^{2}}{4(\alpha+\beta) q \tau_{0}} N^{2}
$$

The parameters $\alpha, \beta$ and $q$ depend on the discharge conditions. For an e-beam sustained system they will depend on the e-beam current that compensates for the decay of metastable Xe atoms.

The efficiency at maximum power defined as $\eta=W_{\max } / P_{\text {opt }}$ is given by:

$$
\eta=\frac{h v}{2}\left\{\alpha-(\alpha+\beta) p \tau_{0}\right\}
$$

which is half its value for low power excitation. The fractional power deposition into the upper state is expressed by $\alpha$ which depends among others on the conductivity of the discharge plasma. The conductivity is strongly influenced by the applied preionization technique or discharge sustainer like the e-beam. The role played by the e-beam, for instance, is basically to produce the initial ion density of the discharge and to compensate the loss of Xe (6s) metastables through the relaxation to the ground level of the atom, so that the metastable level continues to the ground level for the laser. Since the ion density and thereby the conductivity depends on the e-beam current 
intensity it is expected that for a higher e-beam current less power of discharge is used for ionization so that a higher fraction of the input power will be transferred to the upper laser state. This means that $\alpha$ increases with the e-beam current density. One may expect that for the e-beam sustained system the efficiency increases with the e-beam intensity. This has indeed been observed. For x-ray preionized or sustained systems where the degree of ionization is in general much smaller than in the case of e-beam ionization the main discharge adjust itself at a higher $E / N$-value which implies higher average electron energy. More power of discharge is then needed to sustain the discharge and more power is transferred to higher lying states so that the efficiency of the laser is less than in the case of e-beam sustaining. This is confirmed by experiments. ${ }^{(4)}$

\section{EXPERIMENTS}

The experimental parametric studies of the Ar-Xe recombination laser have been carried out with the instrumentation described in Refs $(7,10)$. It is an e-beam sustained system. The e-beam electrons are accelerated in a vacuum diode by a high voltage pulse, up to $270 \mathrm{keV}$, obtained with a Marx generator and enters the laser head through a $25 \mu \mathrm{m}$ thin Ti foil. The sustainer circuit for the main discharge consists of a capacitor of $5.4 \mu \mathrm{F}$ in series with a resistor of about $0.05 \Omega$ to control the current. The energy of this capacitor, charged in the range between 3 and $12 \mathrm{kV}$, is swithced into the discharge by the e-beam. Although the e-beam pulse and sustainer discharge pulse have different durations it is observed that during the simultaneous presence of both pulses strong quasi-stationary laser action is obtained with powers that depend on e-beam current. After the termination of the e-beam pulse having a duration of $1.2 \mu \mathrm{s}$ the laser action continues during the few microseconds that the sustainer pulse is still present, but at a considerably lower level. The power density measurements during the quasi-stationary period have been obtained as a function of gas density up to 9 bar.

A typical example of the observed output power density vs input power density is given in Fig. 3 . The gas mixture contains $0.4 \%$ Xe in 8 bar Ar. The e-beam current is $0.8 \mathrm{~A} / \mathrm{cm}^{2}$ with an energy of $180 \mathrm{keV}$ measured directly behind the transmission foil. ${ }^{(7)}$ The sustainer capacitor is either $1.8 \mu \mathrm{F}$ $(\diamond)$ or $5.4 \mu \mathrm{F}(\bullet)$. As expected the behaviour of the laser depends on the input power density

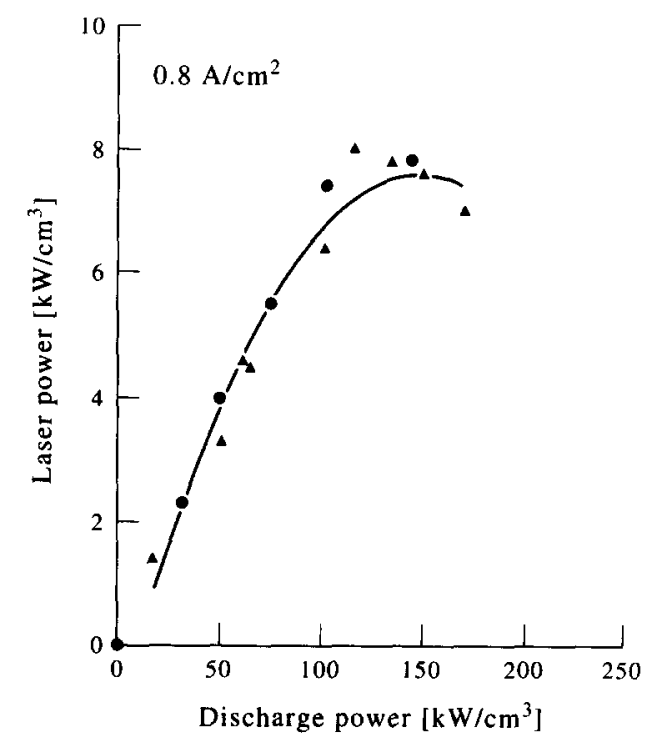

Fig. 3. Output density of a 8 bar e-beam sustained Ar-Xe laser vs input power density. The e-beam current density is $0.8 \mathrm{~A} / \mathrm{cm}^{2}$ and the energy $180 \mathrm{keV}$. The sustainer circuit contains a serial resistor $R_{\mathrm{s}}$ of $0.1 \Omega$ and a capacitor $C_{\mathrm{s}}$ of $1.8 \mu \mathrm{F}(\bullet)$ and $5.4 \mu \mathrm{F}(\mathbf{O})$. 


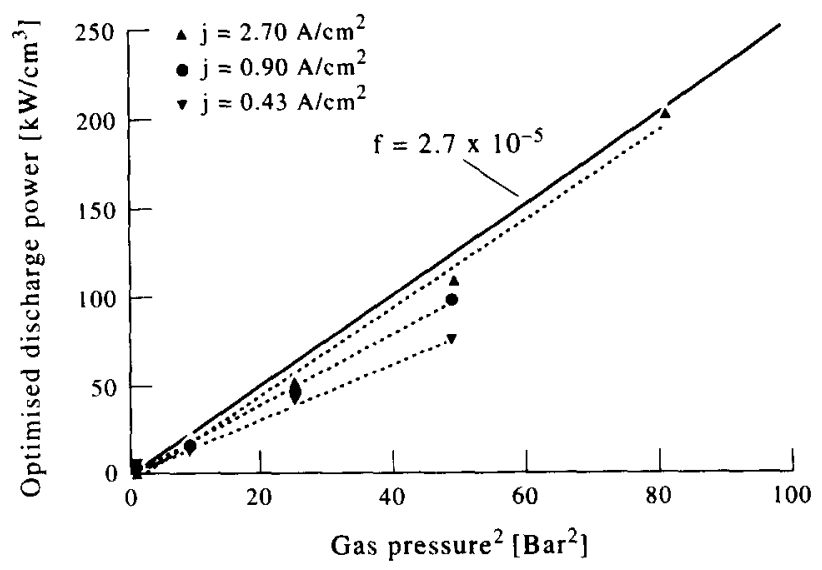

Fig. 4. The observed optimized input power densities vs the square of gas densities for different e-beam current densities.

and not on the capacitor or pulse duration. Under present conditions an optimum input power of about $150 \mathrm{~kW} / \mathrm{cm}^{3}$ is found, independent of capacitor value and pulse duration. Also the predicted curve according to equation (6) is plotted in Fig. 3. Further we plotted in Figs 4 and 5 the observed values for $P_{\text {opt }}$, respectively $W_{\max }$ vs the square of the gas density for various values of the e-beam current. The figures show the more or less linear dependence on the square of the gas density as predicted by (7) and (8). The slopes of the lines depend on $\alpha, \beta$ and $q$, i.e. the average electron energy which in its turn depends on the e-beam current. It is seen that higher efficiencies are indeed obtained with higher e-beam current densities. If the e-beam current or sustainer current becomes too high or the gas density too low then electron quenching will terminate for a short period the laser process after which it starts at low power. ${ }^{(10)}$

In the past there was experimental evidence that the optimized discharge current depended on the gas density. From this it was concluded that there exists an optimum electron density for the active medium of the Xe laser proportional to the gas density or $(n / N)_{\mathrm{opt}}$ is a constant ${ }^{(8-10)}$ This constant was called the fractional ionization. This quantity can also be deduced from the above analysis. This is seen as follows. The electric field of the discharge is proportional to $N$ or $E=N E_{0}$. The current density is given by $i=n e v_{\mathrm{d}}$ where $n$ is the electron density and $v_{\mathrm{d}}$ is drift velocity which depends on $E / N$ and is independent on $N$. The optimized input power density is then given by:

$$
P_{\mathrm{opt}}=N E_{0} v_{\mathrm{D}} e n_{\mathrm{opt}}
$$

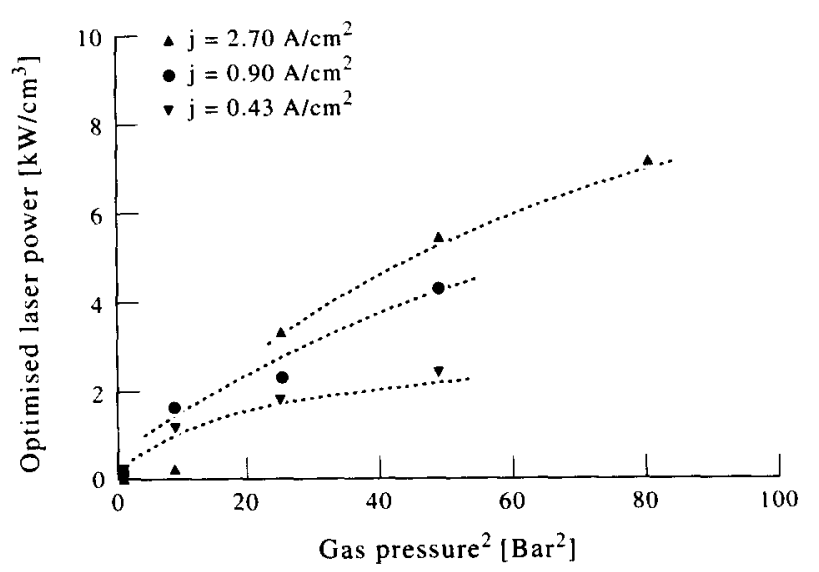

Fig. 5. The observed optimized output power densities vs the square of the gas densities for different e-beam current densities. 
Substituting the last expression into (7) we obtain for the optimum value of $n / N$ or fractional ionization:

$$
\left(\frac{n}{N}\right)_{\mathrm{opt}}=\frac{\left\{\alpha-(\alpha+\beta) p \tau_{0}\right\}}{2(\alpha+\beta) q \tau_{0}} \frac{1}{E_{0} e v_{\mathrm{D}}} .
$$

The right hand side of (11) does not depend on $n$ and $N$ so that an optimum value for $f=n / N$ exists. It is seen that this value depends on $\alpha, \beta, q$ and $E_{0}$ and therefore on the electron energy. However, one may expect that $\beta$ is much smaller than $\alpha$ because the excitation of the laser occurs by recombination and is optimized with respect to $\alpha$. This means that the fractional ionization depends mainly on $E_{0}$ and $q$ and is more or less independent of $\alpha$ and $\beta$. For that reason different values of $f$ are found under differential conditions. For pure e-beam pumping a $f$-value of $2-8 \times 10^{-6}$ is found. ${ }^{(11)}$ For an e-beam sustained system with an e-beam current of $2.7 \mathrm{~A} / \mathrm{cm}^{2}$ the $f$-value can be deduced as follows. At a pressure of 9 bar the observed electrode voltage is $1.83 \mathrm{kV}$ at the electrode separation distance of $2 \mathrm{~cm}$, resulting in $E / N=0.41 \times 10^{-17} \mathrm{~V} / \mathrm{cm}^{2}$. The current density at optimum performance is $217 \mathrm{~A} / \mathrm{cm}^{2}$. The corresponding drift velocity $v_{\mathrm{D}}$ is $2.3 \times 10^{5} \mathrm{~cm} / \mathrm{s}^{\left({ }^{(2)}\right)}$ From this we find $n=6 \times 10^{15} / \mathrm{cm}^{3}$ so that $f=2.7 \times 10^{-5}$. Similarly it can be deduced from Fig. 4 that for smaller values of the e-beam current smaller $f$-values are obtained. This is consistent with equation (11) because for smaller e-beam currents larger $E_{0}$-values are observed. In the case of X-ray preionized self sustained discharges the $E_{0}$-value is even much larger and consequently the $f$-value is much smaller.

We conclude from Fig. 4 that the optimized quasi-steady state input power of the atomic Xe recombination laser is, depending on $E_{0}$, about $1-2.5\left(\mathrm{~kW} / \mathrm{cm}^{-3} / \mathrm{atm}^{-2}\right)$. For a continuous operating system at pressure of 100 torr, which is roughly the minimum pressure for three-bodycollisions, the input power will be about $20-50 \mathrm{~W} / \mathrm{cm}^{3}$. As discussed above, this power density has been successfully transferred into a waveguide slab configuration yielding a continuous output of $1.5 \mathrm{~W}$.

Acknowledgement-These investigations in the program of the Foundation for Fundamental Research on Matter (FOM) have been supported by the Netherlands Technology Foundation (STW).

\section{REFERENCES}

I. N. G. Basov, V. V. Baranov, A. Y. Chugunov, V. A. Danilychev, A. Y. Dudin, I. V. Kholin, N. N. Ustinovskii and D. A. Zayarnyi, IEEE J. Quantum Electron. 21, 1756 (1985).

2. D. E. Rothe and K. V. Tan, Appl. Phys. Lett. 30, 152 (1977).

3. J. E. Tucker, B. L. Wexler, B. J. Feldmann and T. McClelland, IEEE Phot. Technol. Lett. 1, 193 (1989).

4. J. E. Tucker and B. L. Wexler, IEEE J. Quantum Electron. 26, 1647 (1990).

5. L. A. Newman and T. A. De Temple, Appl. Phys. Lett. 27, 678 (1975).

6. Y, B. Udalov, P. J. M. Peters, M. B. Heeman-Ilieva, F. H. J. Ernst, V. N. Ochkin and W. J. Witteman, Appl. Phys. Lett. 63, 721 (1993).

7. H. Botma, P. J. M. Peters and W. J. Witteman, Appl. Phys. B. 52, 277 (1991).

8. M. Ohwa, T. J. Moratz and M. J. Kusher, J. Appl. Phys. 66, 5131 (1989).

9. V. Yu, Baranov, I. M. Isakov, A. G. Leonov, D. D. Malyuta, I. V. Novobrantser, Yu. B. Smakovskii and A. P. Streltsov, Sov. Technol. Phys. Lett. 9, 483 (1983).

10. H. Botma, P. J. M. Peters and W. J. Witteman, IEEE J. Quantum Electron. 29, 2519 (1993).

11. P. J. M. Peters, Y. F. Lan, M. Ohwa and M. J. Kushner, IEEE J. Quantum Electron. 26, 1964 (1990).

12. E. Richley, J. Appl. Phys. 71, 4190 (1992). 